\title{
L'Inde comme expérience de traduction : Olivier Lacombe, René Guénon, René Daumal
}

Il y a bien des manières d'entrer en contact avec des cultures étrangères et, dans ce domaine, le voyage ne garantit rien. On peut parcourir un pays et ne voir que ce que l'on connaît ou croit déjà connaître ; on peut rester lire dans son bureau et se laisser au contraire surprendre par une étrangeté que l'on ne soupçonnait pas. Question de libido, sans doute : comprendre et dominer, d'un côté - parfois avec trop d'empressement ; de l'autre, être saisi et consentir à perdre, au moins pour un temps, ses repères. En tant que chercheur censé trouver quelque chose et parvenir à des résultats, il peut sembler paradoxal d'accepter, voire de favoriser une forme d'égarement face au monde et au texte : un état qui ne serait pas seulement initial mais principiel, qui ne se trouverait nullement écarté à la fin de l'investigation mais qui programmerait sa relance potentiellement infinie. On souhaite ici établir la pertinence d'une défamiliarisation qui ne viendrait pas se résoudre en quelque familiarité que ce soit, quand bien même on ne serait pas exactement dans le même état au début et à la fin du processus de recherche. Il s'agirait de passer d'un état d'ignorance au moins relative à un dessaisissement consenti. On n'aurait pas fait du surplace, il y aurait bien mouvement, mais l'on aurait moins acquis une quantité de savoir qu'établi un certain rapport aux limites du savoir. De ce point de vue, si l'on veut bien admettre que tout objet que l'on aborde dans une optique de connaissance est d'abord un objet étranger que l'on cherche à rendre familier, le contact avec une langue et une culture étrangères fait figure de paradigme. Et plus cette langue et cette culture sont ou semblent étrangères à celui qui l'aborde, plus elles le mettent au défi d'une absence de familiarité et posent avec acuité la question de l'établissement possible d'un savoir.

Cette question, un certain nombre d'esprits français l'ont posée dans les années 1920-1930 en s'intéressant aux langues et aux pensées de l'Inde et à leur traduction en français. Deux précisions préalables s'imposent ici. D'abord, on ne cherchera pas à les situer dans le moment des empires coloniaux fragilisés de l'entre-deux-guerres. Cette étude présenterait un grand intérêt concernant l'étude de la dynamique historique de l'orientalisme, entre structure de domination et événement d'une relation à l'autre, mais entraînerait de trop longs développements de type historique. Il s'agira juste 
de saisir leurs pratiques et leurs discours comme des modèles, comme des types, fondés sur un certain usage de la traduction et de la comparaison et qui programment un certain type de rapport à l'autre, à sa langue, à ses savoirs et, indissociablement, un certain rapport à soi. Délaissant les discours ouvertement racistes prétendant identifier clairement la culture indienne dans sa différence et la considérant comme inférieure et dangereuse, on ne considérera ensuite que les discours impliquant d'une manière ou d'une autre une forme de reconnaissance au moins initiale de la culture indienne (ou de ce qu'on imagine qu'elle est) et de son étrangeté. Ces discours sont au nombre de trois qu'incarnent exemplairement les figures d'Olivier Lacombe, de René Guénon et de René Daumal.

\section{LA TRADUCTION AMALGAMANTE : RENDRE L'ÉTRANGER FAMILIER}

Se donnant pour objet d'exposer la doctrine ontologique de deux maîtres hindous, l'indianiste Olivier Lacombe explique en 1937 dans l'introduction de L'Absolu selon le Védânta. Les notions de Brahman et d'Atman dans les systèmes de Çankara et Râmânoudja qu'il est certes «prématuré » de se lancer dans « une analyse comparative des "philosophèmes" de l'Orient et de l'Occident », parce que, « avant de comparer, il est nécessaire de connaître adéquatement les deux termes de la comparaison ${ }^{1} »$. Une certaine ignorance est ici reconnue, qui inviterait à la prudence. Mais l'auteur estime également qu'il faut « rendre immédiatement intelligible au philosophe de chez nous ces monographies parallèles de deux doctrines indiennes jumelles » et qu'à cette fin « une amorce de comparaison » est « nécessaire $»^{2}$. Il entreprend ainsi de traduire les termes philosophiques tels qu'ils apparaissent dans la langue sanscrite en leur trouvant des correspondants dans les philosophies européennes. «C'est donc toute l'histoire de la philosophie européenne que nous avons appelée à notre secours, choisissant ici ou là, selon les nécessités du moment, le ou les thèmes les plus suggestifs ${ }^{3}$ ». Pour saisir les pensées de Çankara ${ }^{4}$ et de Râmânoudja ${ }^{5}$, Olivier Lacombe

1. Olivier Lacombe, L'Absolu selon le Védânta. Les notions de Brahman et d'Atman dans les systèmes de Çankara et Râmânoudja, Paris, Librairie orientaliste Paul Geuthner, 1937, p. 6.

2. Ibid.

3. Ibid., p. 7.

4. Śankkara (IX ${ }^{\mathrm{e}}$ siècle), de Samkara (celui qui crée la félicité, une des épithètes de Siva), est considéré comme l'un des plus grands maîtres spirituels de l'hindouisme. Voir Śamkara, La Quête de l'être, Textes traduits [du sanscrit], choisis et présentés par Michel Angot, Paris, Le Seuil, coll. Points/Voix spirituelles, 2009.

LITTÉRATURE N 184 - DÉCEMBRE 2016

5. Rāmānuja (XI ${ }^{\mathrm{e}}-\mathrm{XII}{ }^{\mathrm{e}}$ siècles) s'oppose à la philosophie de Śamkara. Il enseigne que toutes les manifestations et les qualités, toutes les différences sont irréelles et transitoires (des reflets du Brahman, mais sans existence propre), tandis que Rāmānuja pense qu'elles sont réelles et permanentes (bien que sous le contrôle du Brahman). Voir Anne-Marie Esnoul, Ramanuja et la mystique vishnouite, Paris, Le Seuil, coll. Maîtres spirituels, 1964. 
ne mobilise ainsi pas moins de dix philosophes occidentaux qui sont tour à tour appelés à comparaître, appartenant aux époques et aux écoles les plus variées, d'Aristote à Descartes ou Spinoza. Ici, l'auteur explique que « c'est du côté de la psychologie dynamique d'un Bergson » mais « aussi loin que possible de Taine » qu'il faudrait chercher « un "climat" analogue à celui de la psychologie indienne »; là, il avance que, « comme Platon dans le mythe d'Er le Pamphylien ${ }^{6}$, Çankara aborde [...] le problème du mal sous l'angle de l'innocence divine »; là également, il se demande « si la notion de mal métaphysique, introduite par l'optimisme leibnitzien dans la philosophie occidentale [...] trouve une place dans la synthèse de l'advaita ${ }^{7}$ ». Les philosophes indiens pensent comme les philosophes européens ou différemment d'eux, mais il est un fait qui ne saurait être contesté : ce sont d'authentiques philosophes que l'on peut faire dialoguer avec leurs homologues.

Que dire du geste de comparaison d'Olivier Lacombe, sinon qu'il est extraordinairement ambigu ? Certes, il a d'abord des vertus pédagogiques. Il s'agit de rendre familier un corpus et des pensées qui sont profondément étrangers au lectorat français et, pour ce faire, il n'est sans aucun doute rien de mieux que de ramener l'inconnu au connu et d'évoquer tel ou tel philosophe étudié dans les universités et les lycées français. Ce geste implique également une forme de valorisation de la pensée indienne. Contre un racisme culturel qui voudrait qu'une certaine forme de rationalité, voire la rationalité dans sa totalité, soit réservée à un Occident seul héritier de ce que certains nomment à l'époque « le miracle $\operatorname{grec}^{8}$ », Olivier Lacombe indique en effet que les philosophies orientales n'ont rien à envier à leurs sœurs occidentales. Comme l'a justement rappelé Roger-Pol Droit ${ }^{9}$, reconnaître l'importance ou même simplement l'existence d'une pensée philosophique qui n'est pas ou ne serait pas occidentale n'est un geste courant ni dans les années 1920-1930 ni même aujourd'hui. De ce point de vue, le geste de traduction opéré par Olivier Lacombe se révèle profondément humaniste, puisqu'il postule une universalité pleine et entière de la démarche philosophique. En matière de philosophie, l'Orient indien ne s'est pas mis à l'école de l'Occident, ce qui déjà aurait supposé une disposition d'esprit adéquat. Il a développé de lui-même une réflexion philosophique, ce qui implique une initiative propre. Le développement de la philosophie ne suit pas un

6. Le mythe d'Er, qui évoque le destin des âmes après la mort et leur réincarnation ultérieure, constitue la clôture du Livre X de la République de Platon. Voir Platon, livre X, 614b-621b dans La République, traduction inédite, introduction et notes par Georges Leroux, Paris, Flammarion, coll. GF, 2002, p. 512-522.

7. Olivier Lacombe, L'Absolu selon le Védânta. Les notions de Brahman et d'Atman dans les systèmes de Çankara et Râmânoudja, op. cit., respectivement p. 133 et p. 256.

8. Voir par exemple Henri Berr, En marge de l'histoire universelle, Tome 1, Les Problèmes de l'histoire; Les origines humaines; Les premières civilisations; Le miracle grec; L'aube de la science, Paris, La Renaissance du livre, coll. L'évolution de l'humanité, 1934.

9. Roger-Pol Droit, L'Oubli de l'Inde [1989], Édition augmentée d'une préface, Paris, Le Seuil, coll. Points, 2004. 
schéma diffusionniste, de la Grèce à l'Europe, puis de l'Europe au reste du monde, en particulier par le biais de la colonisation ; il obéit à un schéma polycentré mais fondé sur l'idée d'un esprit humain universel posant ici et là les mêmes questions et apportant des réponses, sinon toujours identiques, au moins comparables.

On ne peut toutefois s'empêcher de penser que cette reconnaissance d'une raison philosophique indienne constitue un amalgame finalement ethnocentrique - et cela au moins pour deux raisons.

D'abord, on a du mal à imaginer que, d'un point de vue strictement doctrinal, cette manière de procéder n'entraine pas des simplifications outrancières. Près de cent ans après, la façon dont procède Oliver Lacombe s'inscrit en effet dans le droit fil de l'approche de Victor Cousin et du cousinisme. Le philosophe célébrait bien l'Inde comme une terre philosophique et il se réjouissait de la découverte récente de ses doctrines. Appliquant toutefois à toute époque et à toute culture la théorie selon laquelle le sensualisme, l'idéalisme, le scepticisme et le mysticisme sont « quatre points de vue qui servent de base à tous les systèmes, qui sont les éléments nécessaires de toute philosophie », il identifiait dans l'Inde philosophique « leur première apparition ${ }^{10} \gg$ et procédait ainsi à une montée en généralité hâtive. Olivier Lacombe ne pousse certes pas aussi loin l'esprit de système, et les rapprochements qu'il opère sont plus éparpillés. Mais chez lui comme chez son prédécesseur, c'est toujours la raison philosophique occidentale qui sert de mesure à la pensée indienne. La pensée d'un philosophe européen estelle jamais identique, même sur tel ou tel point de détail, à celle d'un autre philosophe européen ? Et, a fortiori, celle d'un philosophe indien est-elle jamais exactement redoublée par celle d'un philosophe européen ? Comme la pensée de Victor Cousin, l'approche d'Olivier Lacombe semble pour le moins cavalière et elle conduit à transformer les pensées de Çankara et de Râmânoudja en un patchwork bariolé et éclectique de pensées européennes.

Ensuite, et plus profondément encore, concernant non pas seulement tel ou tel point de doctrine, mais le mode même de son établissement et son but pratique, une telle traduction ne respecte pas la singularité d'une démarche métaphysique qui n'est peut-être pas exactement de type philosophique ou dont il conviendrait au moins d'interroger le statut propre. Çankara et Râmânoudja sont-ils des philosophes au même titre qu'Aristote, Descartes ou Spinoza ? Leur démarche de pensée n'est-elle pas d'un autre type ? Sans basculer nécessairement dans l'opposition entre philosophie occidentale et sagesse orientale, ne peut-on imaginer qu'il y ait, entre l'Europe et l'Inde et en Inde même, comme en Europe d'ailleurs, des manières

52 différentes d'interroger et de décrire le monde, comme sont différentes la

LITTÉRATURE No 184 - DÉCEMBRE 2016

10. Euvres de Victor Cousin, Tome I, Introduction à l'histoire de la philosophie, Bruxelles, Société belge de Librairie, 1840, respectivement p. 152 et p. 167. Le développement sur l'Inde occupe les pages 152 à 167. 
science et la philosophie, ou la théologie et la philosophie ? Cette universalisation de la philosophie repose sur l'a priori selon lequel l'étranger - ici l'Indien - ne pourrait accéder dans nos contrées à une reconnaissance pleine et entière qu'à la condition de penser à la manière d'un Occidental ${ }^{11}$. Ce que l'on peut soupçonner ici, c'est que la raison universelle dont il est question est une raison occidentale, moins au sens où elle poserait certaines questions et apporterait certaines réponses - Olivier Lacombe soulève ainsi à de nombreuses reprises que l'Inde philosophique ne pense pas comme l'Occident philosophique - qu'au sens où elle obéirait à une manière commune d'interroger le monde. Avec Olivier Lacombe, on est sans doute en présence d'une universalisation en régime occidental ou encore d'un occidentalocentrisme qui s'ignore, qui peut même, en toute bonne foi, reconnaître la valeur de l'autre - mais à condition qu'il soit le même. Ce qu'il peut concevoir, c'est que la pensée hautement spéculative de l'Inde développe des concepts ou des notions dont l'Occident n'aurait pas eu l'idée ; ce qu'il n'imagine pas, en revanche, c'est qu'elle puisse ne pas relever de la philosophie et n'en développer pas moins une forme de rationalité dotée de qualités qui ne la rendraient pas moins rigoureuse pour autant.

La traduction amalgamante constitue une appropriation qui fonctionne par assimilation de l'autre à soi. L'altérité de l'autre n'est certes pas stigmatisée ; mais c'est aussi que cette altérité n'en est en définitive pas une. L'altérité indienne est à peine posée qu'elle se trouve réintégrée dans le cercle de la raison familière. La supériorité différentialiste est certes mise de côté, l'autre n'est pas rejeté comme étranger ; mais une forme de contrôle n'en demeure pas moins active. Y a-t-il toutefois dans les deux cas une même confirmation de soi et une même négation de l'altérité ? Pas tout à fait, parce que ces deux négations ne sont pas du même ordre. La posture différentialiste ne nie pas le fait même qu'il y ait un autre mais la valeur de cet autre ; la posture assimilatrice résorbe in fine le fait même de l'altérité. Il y a, là, une affirmation anthropologique de la différence des cultures et un jugement porté sur leur valeur respective ; et, ici, une négation anthropologique de la différence impliquant une neutralisation axiologique. Ce double constat ne va pas sans paradoxe, quand on envisage ses conséquences culturelles et politiques. La négation de toute différence anthropologique fonde une égalité de droit des êtres humains mais elle est en même temps puissamment négatrice des identités culturelles. À partir du moment où l'on

\footnotetext{
11. Il serait erroné de prétendre que tous les indianistes de l'époque mettent sur le même plan métaphysiciens d'Inde et métaphysiciens d'Europe. Concernant la traduction des notions et concepts, Sylvain Lévi, qui domine le champ de la fin du XIX ${ }^{\mathrm{e}}$ siècle à sa mort en 1935, mesure l'écart des idées sous les ressemblances verbales et, plutôt que d'introduire arbitrairement des concepts relevant de la philosophie occidentale, il choisit de traduire un à un tous les éléments d'un mot original sanscrit et il compose ainsi des mots nouveaux (voir Jacques Bacot, «Introduction » dans Memorial Sylvain Lévi [1937], Delhi, Motilal Banarsidass Publishers Private Limited, coll. Landmarks in Indology, 1996, p. VI-VII).
}

\section{3}

LITTÉRATURE

N 184 - DÉCEMBRE 2016 
prétend que l'humanité est une partout et depuis toujours, il n'y a pas de raison pour changer le paradigme rationaliste qui fonde la reconnaissance de l'autre et l'occidentalocentrisme impose silencieusement son règne. L'autre indien est un familier, mais on le fait entrer de force dans la famille et on le soumet à des exigences qu'on prétend humaines quand elles sont surtout occidentales. Obéissant à une construction de type orientaliste, la dévalorisation de certains groupes humains considérés comme différents peut $a$ contrario conduire à de redoutables conséquences politiques en légitimant des faits de domination, mais elle implique en même temps une reconnaissance de l'altérité dont le signe peut s'inverser. À partir du moment où l'on constate des modes de pensée différents dans différentes cultures, rien n'empêche de transformer le jugement que l'on porte sur elles. On a donc, d'un côté, la stabilité d'une reconnaissance humaniste fondée sur l'absence d'altérité profonde des cultures humaines, et, de l'autre, l'instabilité d'une reconnaissance effective de l'altérité pouvant conduire à sa stigmatisation mais aussi à sa valorisation.

\section{DE LA TRADUCTION IMPOSSIBLE À LA TRADUCTION SYMÉTRIQUE : DÉFAMILIARISER EN RÉTORSION}

Cette démarche de valorisation de l'altérité fondée sur l'absence de familiarité entre les cultures humaines est celle de René Guénon dans l'ensemble des ouvrages qu'il consacre à la tradition hindoue dans les années 1920-1930. À ses yeux, en effet, comme il l'écrit en 1921 dans son Introduction générale à l'étude des doctrines hindoues, l'Occident et l'Orient sont radicalement différents l'un de l'autre et les orientalistes professionnels commettent donc une grave erreur en cherchant à produire « une traduction [...] scrupuleusement littérale ${ }^{12}$ » des textes hindous.

Il n'y a pas d'équivalence véritable entre les termes de deux langues différentes, surtout quand ces langues sont fort éloignées l'une de l'autre, et éloignées non pas tant encore philologiquement qu'en raison de la diversité des conceptions des peuples qui les emploient ; et c'est ce dernier élément qu'aucune érudition ne permettra jamais de pénétrer ${ }^{13}$.

Selon Guénon, le problème de l'absence de termes exactement correspondant est général quand on désire passer d'une langue à l'autre ; il est encore avivé concernant l'Orient et l'Occident dont les mentalités, l'une foncièrement métaphysique, l'autre étrangère à la métaphysique, n'ont rien à voir et dont les langues sont par conséquent très différentes; et il atteint son sommet quand la philosophie occidentale prétend se livrer à l'étude de 
la métaphysique hindoue, alors qu'elle est en fait rigoureusement incapable de le faire avec les outils conceptuels qui sont les siens.

L'Introduction générale à l'étude des doctrines hindoues donne plusieurs exemples des erreurs que suscite « une des manies communes des orientalistes [qui] est de vouloir à toute force faire rentrer la pensée orientale dans ces cadres étroits qui ne sont point faits pour elle ${ }^{14} »$. Ainsi, on discute en Europe du spiritualisme et du matérialisme, alors que la seule authentique conception métaphysique envisageable est celle du « "non-dualisme" » ou encore la « "doctrine de la non-dualité" », traduction approximative, comme l'indiquent les guillemets, du « terme sanskrit adwaita-vâda, qui n'a d'équivalent usuel dans aucune langue européenne » et qui en particulier n'est pas réductible au monisme, «en ce qu'il ne prétend aucunement [...] que l'un des deux termes soit purement et simplement réductible à l'autre ; il les envisage l'un et l'autre simultanément dans l'unité d'un principe commun, d'ordre plus universel, [...] non plus comme opposés mais comme complémentaires ${ }^{15} \gg$. Tout Européen qui s'intéresse aux doctrines hindoues est ainsi confronté à leur opacité, et une opacité fondatrice, qui n'est pas destinée à s'éclaircir par un travail de traduction et de commentaire, mais dont il faut considérer qu'elle demeure tant qu'on cherche à les percevoir dans des cadres qui leur demeurent étrangers.

Ce qu'il faut, c'est tenir un discours à partir de leur propre point de vue, c'est-à-dire penser comme elles, non pas seulement au sens faible où l'on penserait la même chose qu'elles, mais au sens bien plus fort où l'on penserait à leur manière, selon leurs procédures particulières, dans un certain état de l'esprit. Compte moins ici le contenu de la pensée (les doctrines hindoues) que le processus même de la pensée (la manière hindoue de penser les doctrines). Le renversement est même complet quand, rompant radicalement avec tout ethnocentrisme, Guénon met finalement en cause les cadres même de la pensée occidentale par des opérations de traduction inversées et rectificatrices particulièrement puissantes. Ce n'est plus l'Occident qui traduit l'Orient et qui donne sens à ce qu'il pense, mais inversement les catégories de la pensée hindoue qui permettent de saisir une réalité à laquelle l'Occident n'a pas accès par ses philosophies propres ou sa manière propre de philosopher. Guénon dénonce ainsi la notion d'individu comme une pensée typiquement occidentale impliquant également toute une manière illusoire de penser le fait même de penser. Il reprend à son compte l'idée d'un temps cyclique déjà acclimatée en Europe par Nietzsche et il substitue aux philosophies occidentales de l'histoire prétendant décrire les processus historiques depuis Vico et jusqu'à Hegel ou Comte les quatre âges d'un 
obscurcissement graduel de la spiritualité primordiale jusqu' au Kali-Yuga ${ }^{16}$ actuel. Mais l'exemple sans doute le plus frappant de sa démarche est la manière dont il écarte les classifications sociales élaborées en Occident au profit d'une classification d'origine hindoue.

Guénon propose ainsi une description sociale globale et une interprétation de l'histoire, non seulement de l'Inde, mais plus largement de l'Europe, voire du monde, à la lumière des quatre castes traditionnelles de l'Inde, les Brâhmanes, les Kshatriyas, les Vaishyas et les Shûdras ${ }^{17}$. L'auteur prévient les critiques qu'on pourrait lui adresser :

On nous reprochera peut-être de parler comme s'il y avait des castes partout, et d'étendre indûment à toute organisation sociale des dénominations qui ne conviennent proprement qu'à celle de l'Inde ; et pourtant, puisque ces dénominations désignent en somme des fonctions qui se retrouvent nécessairement dans toute société, nous ne pensons pas que cette extension soit abusive ${ }^{18}$.

Les castes désignent en effet des fonctions sociales qui témoignent de différences de nature et d'aptitude entre les individus, fonctions et individus qui existent dans tous les groupes humains. À partir de ces considérations, on peut opposer deux types de sociétés : celles qui sont structurées par le système des castes et dans lesquelles on observe « une correspondance normale entre la nature des individus et les fonctions qu'ils exercent », et celles dans lesquelles « cette correspondance n'existe pas, ou, du moins, ne se rencontre qu'accidentellement ${ }^{19}$. C'est pour expliquer la disparition de l'ordre traditionnel qui caractérise l'Inde mais que l'Europe a oublié que Guénon considère la lutte des castes comme le moteur de l'histoire. De la querelle du Sacerdoce et de l'Empire au Moyen Âge à la révolution de 1917, c'est l'anarchie qui, de fil en aiguille, s'est partout répandue. À une chrétienté dominée par un empereur lui-même soumis au pape succèdent des monarchies européennes puis des nations en guerre, tandis que l'unité de la chrétienté est détruite par l'individualisme protestant et que les révolutions achèvent l'asservissement de l'autorité spirituelle à des pouvoirs temporels de plus en plus dégradés. Les Brâhmanes laissent peu à peu la place aux Kshatriyas (les monarques), les Kshatriyas aux Vaishyas (les bourgeois) avec le triomphe de l'idéologie du progrès et du bien-être matériel, et finalement les Vaishyas aux Shûdras (le peuple) avec la victoire du bolchevisme -

16. Le Kali-Yuga est le quatrième âge de la cosmogonie hindoue, dans lequel nous vivons actuellement. Les hindous croient que la civilisation humaine dégénère spirituellement au cours du Kali-Yuga et que c'est donc l'âge lors duquel les êtres sont les plus nombreux à souffrir. 17. Les quatre varnas hindous tirés des textes védiques sont le plus souvent traduits par le terme de castes : ce sont les brāhmana (liés au sacré), prêtres, enseignants et professeurs ; les ksatriya (qui ont le pouvoir temporel), roi, princes, administrateurs et soldats ; les vaiśya (liés au clan), artisans, commerçants, hommes d'affaires, agriculteurs et bergers ; et les śūdra (serviteurs), qui servent les précédents. Voir Robert Deliège, Le Système indien des castes, Villeneuve-d'Ascq, Presses universitaires du Septentrion, coll. Les savoirs mieux, 2006.

LITTÉRATURE 18. René Guénon, Autorité spirituelle et Pouvoir temporel, Paris, Vrin, 1929, p. 56. 
véritable «nuit intellectuelle » d'une « ignorance qui se prend pour science et se complaît dans son néant ${ }^{20} »$.

Une telle lecture de l'organisation de la société occidentale et de sa dégénérescence n'est certes pas familière au lecteur occidental ; et pas plus aujourd'hui qu'en 1929. Il s'agit d'un conflit de catégories de la construction de la réalité qui pose la question plus large de la légitimité de l'application de catégories exogènes à une réalité sociale ou culturelle donnée : les Hindous pensent leur réalité sociale et individuelle en termes de castes ; les Européens auraient plutôt tendance à la penser en termes de classes (ou d'individus). Mais peut-on lire l'Inde hindoue en termes de classes, et l'Europe capitaliste ou communiste en termes de castes ? Du point de vue d'un Européen, la seconde démarche semble problématique, alors que la première ne fait pas problème. Même si l'on ne se prononce pas sur le fond de la question, le geste de rétorsion opéré par Guénon invite à deux réflexions que prolonge aujourd'hui à sa manière Dipesh Chakrabarty dans Provincialiser l'Europe lorsqu'il se demande si « le marxisme » est « tout simplement "vrai" » ou s'il est « une manière provisoire d'organiser une masse d'informations » liée à ses « racines européennes ${ }^{21}$ ». D'abord, ce geste indique en miroir la violence de l'Occident quand il applique ses catégories indigènes à des cultures étrangères pour lesquelles elles ne signifient rien ou pas grand-chose. Si l'Occident entend déchiffrer l'Orient, pourquoi l'Orient ne pourrait-il faire de même, non pas avec les outils de l'Occident, dans une perspective critique préservant toutefois les mêmes catégories de pensée (critique par exemple de l'impérialisme occidental dans une perspective marxiste), mais plus profondément encore, avec ses propres outils de pensée ? Ce que Guénon nous pousse à imaginer, c'est une mondialisation en régime hindou, mieux encore une domination symbolique mondiale de la pensée hindoue comparable à celle qu'exerce effectivement la pensée occidentale. Il nous invite également à défamiliariser nos propres catégories et nos propres modalités de pensée. Ce n'est pas l'Hindou qui est étranger aux yeux de l'Occidental, c'est l'Occidental qui devient un étranger aux yeux de l'Hindou. Et dans cette inversion du point de référence, il convient, sinon, comme le souhaitait Guénon, de tout apprendre ou réapprendre de l'Orient, au moins de mesurer la relativité de nos propres pensées et des systèmes de raison qui les organisent. Ce que nous percevons comme naturel ne l'est nullement et constitue simplement une construction possible de la réalité dont la validité doit être précisément mesurée.

Que cette démarche n'aille pas elle aussi sans aporie, il faut toutefois le relever. Trois d'entre elles s'imposent tout particulièrement à l'esprit concernant le point précis des relations entre les cultures et la question de la

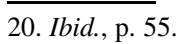

21. Dipesh Chakrabarty, « Préface à l'édition de 2007 », Provincialiser l'Europe. La pensée postcoloniale et la difference historique [2000], Paris, Éditions Amsterdam, 2009, p. 18.

LITTÉRATURE

Nº184 - DÉCEMBRE 2016 
familiarité. D'abord - et de ce problème central découlent les deux autres -, même s'il pense la différence entre Orient et Occident comme le fruit d'un processus historique, Guénon n'en a d'abord pas moins tendance à figer les deux ensembles comme absolument antithétiques et il néglige leur pluralité interne autant que les échanges qu'ils peuvent avoir l'un avec l'autre. En considérant que la valeur ne se situe pas du côté de la modernité et que la vectorisation historique occidentale est une vue déformée de la réalité, Guénon ne fait ensuite que renverser le diagnostic en considérant que ce n'est plus l'Orient qui est en retard sur l'Occident, mais que c'est ce dernier qui constitue une dégénérescence par rapport à un Orient dépositaire de la tradition. De ce point de vue, il pense encore les civilisations en termes de hiérarchie et il ne fait que substituer au mépris orientaliste de l'Occident pour l'Orient une sorte d'occidentalisme tout aussi totalisant. Enfin, opposant Orient et Occident comme deux entités essentiellement différentes, Guénon a le plus grand mal à penser comment, malgré le souhait ardent qui est le sien, l'Occident pourrait s'orientaliser. Cette position ne va en outre pas sans une contradiction majeure. Ce n'est pas seulement que l'Occidental Guénon s'arroge l'accès à la métaphysique orientale tout en prétendant que ses contemporains d'Occident sont incapables de la saisir. C'est surtout qu'on ne comprend pas bien comment la traduction impossible de notions sanscrites dans les langues européennes autorise au rebours une traduction de notions européennes dans des catégories de la langue sanscrite. C'est que le point d'où est formulé le discours change ici du tout au tout : ce n'est plus depuis la raison philosophique occidentale, comme chez Olivier Lacombe, qu'est formulé le discours de la familiarisation, mais depuis la pensée indienne (telle que l'imagine un Occidental toutefois). En somme, avec Guénon, l'Inde reste étrangère à l'Occident, elle lui demeure impénétrable, mais l'Occident se laisse déchiffrer par l'Inde (ou plutôt par son porte-parole privilégié qu'est Guénon lui-même) et se trouve dès lors amalgamé à sa pensée.

\section{LA TRADUCTION APPROXIMATIVE : MAINTENIR UNE DOUBLE ÉTRANGETÉ}

Avec Lacombe, on tombe dans le piège d'une traductibilité intégrale qui rend absolument familier ce qui était censé ne pas l'être, et toute altérité se trouve niée ; avec Guénon, l'altérité est bien reconnue, elle est même valorisée, mais c'est alors ce qui était censé être familier qui se trouve pris au piège de la pensée de l'autre et devient dès lors étranger à soimême. Dans le premier cas, l'Inde intègre de force la grande famille des hommes qui se trouve être en fait la petite famille occidentale; dans le second, elle forme une famille apparemment spécifique mais qui se trouve être celle de tous les hommes et qui absorbe tout dans son empire de 
pensée. Sortir de ce piège symétrique d'un excès ou d'une absence de familiarité, d'une défamiliarisation de l'autre réduite par l'universalisme à une défamiliarisation de soi par la pensée de l'autre, suppose de faire la part du différent et du commun tout en maintenant une certaine opacité : d'accepter un accès limité, non pas d'abord mais in fine, à l'autre comme à soi-même. Cette démarche est à l'occasion celle de René Daumal dans les multiples textes qu'il consacre à l'Inde des années 1920 à sa mort en 1944.

Certes Daumal n'évite pas toujours un certain éclectisme et il lui arrive de mentionner, au milieu d'ouvrages hindouistes, d'autres sans aucun rapport avec l'Inde ou la sphère d'influence indienne, qu'il s'agisse d'ouvrages de William James, de Milosz, de Pascal ou encore des visions d'Ezéchiel et de saint Jean ${ }^{22}$. À côté des références à la mystique chrétienne, Daumal emprunte plus largement à toutes sortes de traditions non occidentales : kabbale juive, mystique soufie, bouddhisme tibétain, taoïsme chinois ou zen japonais. Cette soif de connaissance conduit à des rapprochements encore plus larges que ceux opérés par Lacombe et elle produit des chaînes d'équivalence sans aucun doute abusives, puisqu'elles reviennent à prétendre que tout est dans tout et que les savoirs et les pratiques venus d'horizons culturels différents sont finalement les mêmes. De ce point de vue, l'autodidacte qu'est Daumal n'échappe pas toujours, surtout dans ses années de jeunesse, au raccourci rapide et au fourre-tout spirituel de l'Occidental cosmopolite venant se servir librement dans le répertoire des croyances du monde. Mais sa démarche va aussi bien au-delà. Non seulement c'est vers l'Inde qu'il revient le plus souvent, mais c'est l'Inde qui devient le point de référence central à partir duquel il pense le monde et évalue les autres pensées.

Profondément influencé par la lecture des œuvres de Guénon dont il reprend à maintes reprises les analyses concernant la décadence de «l'Occident individualiste-dualiste-libre-arbritiste, triste, capitaliste-colonialisteimpérialiste ${ }^{23} \gg$ et la valorisation en contraste de la spiritualité orientale comme connaissance en action, Daumal entreprend lui aussi une critique des savoirs orientalistes aveuglés par leurs propres présupposés et il conduit une entreprise de dénaturalisation des notions occidentales les mieux admises, en particulier les notions d'individu créateur et de poésie, dont il explique à partir des textes hindous qu'elles sont en fait inaptes à décrire ce qu'est la créativité. Pour passionnante qu'elle soit, ce n'est toutefois pas cette entreprise guénonienne de déconstruction conduisant à défamiliariser et à périphériser l'Occident au profit d'un savoir qui lui est étranger et supérieur qui sera développée ici, mais un point plus réduit qui n’en est pas

22. René Daumal, « Le souvenir déterminant », Les Pouvoirs de la parole. Essais et notes II (1935-1943), Paris, Gallimard, 1972, respectivement p. 112, p. 114 et p. 119.

23. René Daumal, « À propos d'Uday Shankar et de quelques autres hindous » [1932], L'Évidence absurde. Essais et notes, I (1926-1934), Paris, Gallimard, 1972, p. 255. 
moins d'une grande portée : la manière dont Daumal pratique et envisage la traduction du sanscrit en français.

Daumal méprise les intellectuels et les écrivains français qui n'hésitent pas à énoncer de grandes vérités sur l'Inde sans avoir une bien grande connaissance des textes ou qui, pire encore, sont persuadés d'avoir percé leur étrangeté. Ce qu'un savant orientaliste peut faire de mieux à ses yeux, c'est traduire un texte le plus littéralement qui soit sans ajouter ni introduction philosophique, ni commentaire qui ne manqueraient pas de fausser son sens. Dans le cas contraire, c'est le contresens presque assuré :

Il faut se méfier des livres religieux et philosophiques de l'Orient comme de la peste. Sauf si vous les lisez dans le texte. Autrement, vous avez le choix entre la traduction du savant linguiste qui transforme tout en platitudes, et celle, puante, du théosophe qui sait tout par la révélation du petit bout de bois et vous sort, dans une pâte de mauvais anglais ou français, des mots palis à désinences sanscrites ou des mots tibétains à la sauce métaphysique de l'Intelligence Service d'Adyar ${ }^{24}$.

Accéder à la source authentique de l'Inde par le biais de la lecture des textes originaux est une priorité pour Daumal, qui n'entend pas se laisser tromper sur la marchandise par des truchements occidentaux ou indiens qui dénatureraient par ignorance ou opportunisme la pureté du message de l'Inde. Tel est le principe de sa critique des orientalistes mais aussi d'un Krishnamurti sous l'emprise des « infamies spirituelles ${ }^{25}$ » de la théosophie ou encore de tel ou tel danseur indien qui, comme Rao ou Indra Ramosay, proposent de «prétendues danses "sacrées" [qui] sont de bien misérables caricatures $»^{26}$. Ne perdant jamais la conscience de l'étrangeté du texte à commenter, lui-même se montre d'une extrême prudence interprétative et ne cesse de critiquer, non seulement les travaux des autres, mais surtout ses propres travaux de traduction. Deux points retiennent son attention. D'abord, toute traduction est limitée du fait de la polysémie du mot (sanscrit), polysémie qu'on ne peut rendre que dans une paraphrase. Ensuite et surtout, cette paraphrase elle-même est impossible à établir dans une version unique, puisqu'elle dépend de la personne du traducteur et de son état intérieur : de ce que, à qu'un moment donné, il est capable de comprendre de ce qu'il traduit. Le geste de traduction est ainsi soumis à deux conditions qui

24. René Daumal, « Le Livre des Morts Tibétain » [1934], Les Pouvoirs de la parole. Essais et notes II (1935-1943), op. cit., p. 175. Les éditions Adyar, qui existent encore aujourd'hui, ont pour objet de faire connaître l'enseignement théosophique. Voir http://editionsadyar.com/ 25. René Daumal, lettre à Carlo Suarès [Paris, mi-mai 1932], Correspondance, II 1929-1932, Paris, Gallimard, coll. Les Cahiers de la NRF, 1993, p. 282. D'abord séduit par Krishnamurti lorsqu'il le rencontre en octobre 1930 à Paris, à l'occasion de la réception donnée en son honneur par Carlo Suarès, Daumal se désolidarise de l'un comme de l'autre, à la suite de sa lecture du manuscrit de l'ouvrage de Suarès, Krishnamurti (Paris, Adyar, 1932).

LITTÉRATURE 26. René Daumal, lettre à Émile Dermenghem, 23 août [1934], Correspondance, III 1933-1944, Paris, Gallimard, coll. Les Cahiers de la NRF, 1996, p. 47. 
impliquent un tel déploiement et redéploiement du sens qu'elles rendent impossible l'établissement d'une version totale et définitive.

Voici ce qu'écrit Daumal quand il traduit la première stance du NâtyaÇâstra ${ }^{27}$ : «Tête courbée devant le Grand-père céleste et le céleste Prince, je vais exposer l'Enseignement du Théâtre, qui fut prononcé par Brahmân ${ }^{28} »$. Le commentaire qui suit indique toute la difficulté de la traduction : « Grandpère $=$ Brahmâ. Prince $=$ Çiva. Le maître de la Parole et le maître de la Danse sont les pères de l'art dramatique. Si vous tenez à traduire "Brahmâ", dites "le Proférateur" 29 ». La dernière phrase désinvolte de Daumal le montre bien : toute traduction implique un choix qui est laissé en partie à l'appréciation de chacun et elle ne peut donc parfaitement convenir qu'au traducteur luimême. On voit également le travail sur la multiplication des désignations, qui déjoue l'égalité initiale du signe mathématique. Écrire en effet « Grand-père = Brahmâ » ne saurait suffire, puisque Brahmâ est aussi « le Proférateur » et en outre - mais le lien n'est pas explicite - « le maître de la Parole ». Et il en va de même pour Çiva, à la fois «Prince » et «maitre de la Danse ». Le passage dans la langue française de la totalité des possibles sémantiques contenus dans la sentence hindoue se révèle ainsi impossible et la traduction manque pour partie son objet.

C'est cet affrontement avec les limites du traduisible qui explique l'humilité profonde de Daumal, sa perpétuelle insatisfaction et sa tentation du renoncement à toute entreprise de ce type. Exposant les principes de la poésie hindoue, il se refuse ainsi à donner des exemples (nécessairement mal) traduits du sanscrit : "Je n'ai guère cherché à rendre en français des citations de poèmes sanskrits donnés comme exemples; le lecteur trouvera dans sa propre langue, chez les poètes qu'il aime, des exemples bien plus convaincants que des traductions forcément imparfaites ${ }^{30} »$. Si peu des traductions de Daumal ont paru de son vivant, c'est peut-être l'effet d'une difficulté à finir par excès de perfectionnisme, mais c'est surtout le fruit d'une conviction : chaque traduction ne peut être réellement utile qu'à celui qui la fait, au moment où il la fait. Figer une traduction serait dès lors un non-sens. Si toute traduction témoigne de la lecture d'un homme à un certain moment de sa vie, elle peut être remise en cause par un autre homme ou même lors d'une lecture ultérieure du même homme, et Daumal ne peut dès lors ni arrêter sa traduction en la publiant ni s'arrêter de traduire. Plus d'impérialisme linguistique ici, plus de langue universelle qui pourrait dire

27. Le Nâtya-shâstra, composé par Muni Bharata vers 400 av. J.-C., est un traité encyclopédique qui expose les codifications pour le théâtre, le jeu dramatique, la poésie, la danse, le chant et la musique.

28. René Daumal, «L'origine du théâtre de Bharata » [1935], Bharata. L'origine du théâtre.

La poésie et la musique en Inde, Paris, Gallimard, [1970] 2009, p. 28.

29. Ibid., p. 38.

30. René Daumal, « Les pouvoirs de la parole dans la poétique hindoue » [1938], ibid., note 1 p. 83. 
le monde entier des pensées et leur faire rendre raison, mais des frottements, des intersections - un brouillard qu'on cherche à dissiper.

Y parvient-on jamais ? Convient-il de suivre Daumal jusqu' au bout de son raisonnement, qui le conduit à penser que la compréhension progressive d'un texte sanscrit n'est possible que sous la direction d'un gourou qui permette d'apprendre l'oubli de soi ? Dans un texte de 1941, il note ainsi « la nécessité d'un guide, d'une école », et il revient sur ses années d'errance :

L'erreur que j'avais commise était celle-ci : j'avais interrogé les textes sanskrits comme si j'eusse été moi-même un brāhmane, un sannyāsin, un yogin. [...] Ayant changé la direction de mes recherches et de ma vie, ayant reconnu, par exemple, que les upanishad ne sont pas offertes en pâture à n'importe qui (or, je suis encore un "n'importe qui”" !) mais sont une lumière toujours allumée et que c'est à moi de me faire pousser des yeux pour la voir, par des efforts longs et douloureux, et sous la direction de quelqu'un ayant déjà des yeux - je ne voulais tout de même pas que le temps passé à mes études sanskrites fût perdu ${ }^{31}$.

Il est question ici d'une progression spirituelle accompagnée qui permet peu à peu de comprendre ce qu'on lit, de le comprendre différemment, c'est-à-dire de plus en plus profondément. Comme Guénon, Daumal a ici en tête l'opposition entre ce que la doctrine contient de sens exotérique et ésotérique et le chemin intérieur qui mène de l'un à l'autre. À la fois exemple auquel on s'identifie, professeur qui enseigne et maître qu'on a le privilège de servir, le gourou est une figure bien identifiée en Inde mais inconnue en Occident et il est peu probable qu' on puisse facilement le transposer dans nos habitudes de lecteur. Il est trop lié à la lecture des seuls textes hindous pour pouvoir concerner celles d'autres textes.

Mais l'important est peut-être ailleurs : Daumal maintient la pensée dans cette «pluralité » des langues qu'évoque Barbara Cassin dans sa réflexion sur ce qu'elle nomme « les "intraduisibles" », mots qui certes peuvent être traduits d'une langue à l'autre, mais qui perdent alors (et gagnent aussi) quelque chose en route, ce nœud d'intraductibilité qui oblige à des distorsions répétées, si bien que finalement « on ne cesse pas de (ne pas) traduire ${ }^{32} »$. Le statut d'une telle traduction n'est donc pas de donner au lecteur français accès au texte sanscrit, pas même peut-être de constituer un appas le conduisant à emprunter finalement le chemin de Daumal lui-même vers le texte original. Faire son expérience, c'est reconnaître qu'on ne peut accueillir l'autre qu'imparfaitement dans sa langue, c'est aussi accepter que, quand bien même on accéderait à sa langue à lui, on ne saurait pas davantage parvenir au sens définitif des textes qu'il donne à lire.

31. René Daumal, « Ce que l'Inde m'a appris » [1941], repris dans Claudio Rugafiori, « Préface », ibid., p. 10-11. 
L'expérience de traducteur de Daumal est une expérience que nous faisons tous quand nous traduisons et plus largement encore quand nous lisons ou plutôt quand nous relisons. Telle pourrait être la leçon du gourou daumalien, ou plutôt de son absence occidentale : ce qu'il s'agit de préserver, c'est la part de l'autre: quelque chose qui n'empêche pas d'aller à sa rencontre - l'autre n'est pas l'étranger absolu de l'exotisme - mais qui n'appartient qu'à lui, qui ne peut tout à fait se dire et se penser, même dans sa propre langue, et qui limite donc la prétention, non pas seulement du sujet occidental à le posséder intégralement dans sa langue à lui, mais de tout sujet à posséder quelque texte que ce soit dans un métalangage. Au-delà de la question de la traduction, ce que pose Daumal, c'est ainsi une limite qui est celle de la rencontre avec un texte, de la relation qu'on entretient avec lui, impliquant, non pas de l'impénétrable (les zones de résistance sont mouvantes et se déplacent), mais d'un reste qui n'est pas pénétré, au moins en un moment, à une date donnés et selon des aléas qu'on ne maîtrise pas. La figure du gourou telle que la pense Daumal enseigne ainsi à prendre garde, non seulement au geste même de la traduction, mais à toute lecture comme approximation permanente : contre toute prétention qui garantirait l'autorité de la lecture, ne jamais cesser de se faire pousser des yeux - ce qui revient à dire : ne pas oublier qu'on est borgne, ou presbyte, ou myope (et l'on pourrait ici filer et enrichir les métaphores optiques indiquant qu'on ne voit bien que la moitié des choses, ou seulement l'ensemble qu'elles forment mais pas les détails, ou tel détail aux dépens du tout, etc.). Dans le fond, c'est autant l'Inde qu'il convient de défamiliariser que, à partir de l'Inde, soi-même et ses propres routines de pensée dans une opération réciproque. Chercher non pas ce qu'on croit savoir, rester soi-même ou passer à l'autre, mais errer dans l'entre et faire face à ce qui déroute : une étrangeté qui ne se résorbe pas, une familiarité qui ne s'établit jamais. Non pas l'ineffable, non pas l'inconnu, non pas le mystère ; mais simplement : le maintien d'une étrangeté contre la violence du traduit ${ }^{33}$.

33. L'auteur tient à remercier Tiphaine Samoyault qui lui a donné l'occasion de développer une première ébauche de son propos lors de la journée Défamiliariser qu'elle a organisée à l'Université Paris 3 Sorbonne-nouvelle le 21 juin 2013.

LITTÉRATURE N ${ }^{\circ} 184$ - DÉCEMBRE 2016 